Supporting Information

\title{
General Silaindene Synthesis Based on Intramolecular Reductive Cyclization Toward New Fluorescent Silicon-Containing $\pi$-Electron Materials
}

Caihong Xu, Atsushi Wakamiya, and Shigehiro Yamaguchi*

Department of Chemistry, Graduate School of Science, Nagoya University, and PRESTO, Japan Science and Technology Agency (JST), Chikusa, Nagoya 464-8602, Japan

\section{General Procedure for the Intramolecular Reductive Cyclization.}

Synthesis of 5a. A mixture of granular lithium (28 mg, $4.03 \mathrm{mmol})$ and naphthalene (519 mg, $4.05 \mathrm{mmol})$ in THF (4.5 mL) was stirred at room temperature under argon for $4 \mathrm{~h}$. To the produced solution of lithium naphthalenide was added a solution of compound 4 (396 mg, $1.00 \mathrm{mmol})$ in THF (2 mL) at room temperature. After stirred for $5 \mathrm{~min}$, the reaction mixture was quenched with a saturated aqueous solution of $\mathrm{NH}_{4} \mathrm{Cl}$ at the same temperature. The mixture was extracted with ether for several times and the combined organic layer was washed with brine, dried over $\mathrm{MgSO}_{4}$, filtered, and concentrated under reduced pressure. The resulting mixture was passed through a silica gel column (hexane, $R_{\mathrm{f}}=0.15$ ) and purified by a preparative gel permeation chromatography (eluent: $\left.\mathrm{CHCl}_{3}\right)$ to give $205 \mathrm{mg}(0.52 \mathrm{mmol})$ of $\mathbf{5 a}$ in $52 \%$ yield as a pale yellow solid: mp 203-205 ${ }^{\circ} \mathrm{C} . \quad{ }^{1} \mathrm{H}$ NMR $\left(270 \mathrm{MHz}, \mathrm{CDCl}_{3}\right): \delta 0.50(\mathrm{~s}$, 12H), $7.22(\mathrm{~m}, 2 \mathrm{H}), 7.33(\mathrm{~m}, 4 \mathrm{H}), 7.52(\mathrm{~s}, 4 \mathrm{H}), 7.56(\mathrm{~m}, 4 \mathrm{H}) .{ }^{13} \mathrm{C} \mathrm{NMR}(67.8 \mathrm{MHz}$, $\left.\mathrm{CDCl}_{3}\right): \delta-3.06,124.34,126.64,126.95,130.08,131.73,137.94,138.34,140.82$, 
144.91, 148.99. ${ }^{29} \mathrm{Si}$ NMR (79.3 MHz, $\left.\mathrm{CDCl}_{3}\right): \delta$ 3.92. Anal. Calcd for $\mathrm{C}_{26} \mathrm{H}_{26} \mathrm{Si}_{2}$ : C, 79.13; H, 6.64. Found: C, 79.25; H, 6.67.

\section{Spectral and Analytical Data for Silaindene Derivatives}

2: Mp. 65-67 ${ }^{\circ} \mathrm{C} .{ }^{1} \mathrm{H}$ NMR (400 $\left.\mathrm{MHz}, \mathrm{CDCl}_{3}\right): \delta 0.49(\mathrm{~s}, 6 \mathrm{H}), 7.20-7.28(\mathrm{~m}, 1 \mathrm{H})$, 7.30-7.40 (m, 4H), 7.48-7.58 (m, 4H). $\left.{ }^{13} \mathrm{C} \mathrm{NMR} \mathrm{(100} \mathrm{MHz,} \mathrm{CDCl}_{3}\right): \delta-3.14,124.31$, $126.49,126.64,127.06,128.74,130.03,131.70,138.35,139.11,141.17,145.39,148.88$. ${ }^{29} \mathrm{Si}$ NMR $\left(79.3 \mathrm{MHz}, \mathrm{CDCl}_{3}\right): \delta$ 3.95. HRMS(FAB): $236.1029\left(\mathrm{M}^{+}\right)$. Calcd for $\mathrm{C}_{16} \mathrm{H}_{16} \mathrm{Si}: 236.1021$.

3: Mp. 132-134 ${ }^{\circ} \mathrm{C} . \quad{ }^{1} \mathrm{H}$ NMR $\left(400 \mathrm{MHz}, \mathrm{CDCl}_{3}\right): \delta 0.37(\mathrm{~s}, 6 \mathrm{H}), 0.41(\mathrm{~s}, 6 \mathrm{H}), 6.82-$ $6.90(\mathrm{~m}, 4 \mathrm{H})$, 6.98-7.05 (m, 5H), 7.10-7.15 (m, 2H), 7.20-7.28 (m, 5H), 7.56-7.62 (m, 2H). ${ }^{13} \mathrm{C} \mathrm{NMR}\left(67.8 \mathrm{MHz}, \mathrm{CDCl}_{3}\right): \delta-3.78,-3.29,124.05,125.87,126.66,127.38$, 127.61, 130.04, 131.48, 137.84, 139.92, 144.10, 149.82, 150.51. Anal. Calcd for $\mathrm{C}_{32} \mathrm{H}_{30} \mathrm{Si}_{2}:$ C, 81.64; H, 6.42. Found: C, 81.42; H, 6.32.

5b: Mp. 260-262 ${ }^{\circ} \mathrm{C} . \quad{ }^{1} \mathrm{H}$ NMR $\left(400 \mathrm{MHz}, \mathrm{CDCl}_{3}\right): \delta 0.38$ (s, 12H), $2.21(\mathrm{~s}, 6 \mathrm{H}), 7.18$ (s, 4H), 7.24-7.28 (m, 2H), 7.38-7.44 (m, 4H), 7.57 (m, 2H). ${ }^{13} \mathrm{C}$ NMR (67.8 MHz, $\left.\mathrm{CDCl}_{3}\right): \delta-3.72,14.58,121.73,126.44,128.04,129.84,131.23,138.21,138.27,142.19$, 148.11, 150.94. ${ }^{29} \mathrm{Si} \mathrm{NMR}\left(79.3 \mathrm{MHz}, \mathrm{CDCl}_{3}\right): \delta$ 2.91. Anal. Calcd for $\mathrm{C}_{28} \mathrm{H}_{30} \mathrm{Si}_{2}: \mathrm{C}$, 79.56; H, 7.15. Found: C, 79.32; H, 7.18.

5c: Mp. $182-184{ }^{\circ} \mathrm{C} . \quad{ }^{1} \mathrm{H}$ NMR $\left(400 \mathrm{MHz}, \mathrm{CDCl}_{3}\right): \delta 0.21(\mathrm{~d}, J=3.6 \mathrm{~Hz}, 12 \mathrm{H}), 0.35(\mathrm{~s}$, 12H), $4.38(\mathrm{sep}, J=3.6 \mathrm{~Hz}, 2 \mathrm{H}), 7.06(\mathrm{~s}, 4 \mathrm{H}), 7.23(\mathrm{~m}, 2 \mathrm{H}), 7.39(\mathrm{~m}, 2 \mathrm{H}), 7.58-7.62(\mathrm{~m}$, 4H). ${ }^{13} \mathrm{C} \mathrm{NMR}\left(67.8 \mathrm{MHz}, \mathrm{CDCl}_{3}\right): \delta-4.23,-2.63,124.98,126.01,126.73,129.90$, $131.82,138.28,140.63,152.61,153.80,163.93 .{ }^{29} \mathrm{Si}$ NMR $\left(79.3 \mathrm{MHz}, \mathrm{CDCl}_{3}\right): \delta$ 
-27.36, 4.47. Anal. Calcd for $\mathrm{C}_{30} \mathrm{H}_{38} \mathrm{Si}_{4}: \mathrm{C}, 70.52 ; \mathrm{H}, 7.50$. Found; C, 70.26; H, 7.35.

5d: Mp. 282-284 ${ }^{\circ} \mathrm{C} . \quad{ }^{1} \mathrm{H}$ NMR (400 MHz, $\left.\mathrm{CDCl}_{3}\right): \delta 0.36(\mathrm{~s}, 12 \mathrm{H}), 1.30$ (s, 24H), 7.18 $(\mathrm{t}, J=7.2 \mathrm{~Hz}, 2 \mathrm{H}), 7.26(\mathrm{~s}, 4 \mathrm{H}), 7.34(\mathrm{t}, J=7.2 \mathrm{~Hz}, 2 \mathrm{H}), 7.47(\mathrm{~d}, J=7.2 \mathrm{~Hz}, 2 \mathrm{H}), 7.52$ $(\mathrm{d}, J=7.2 \mathrm{~Hz}, 2 \mathrm{H}) .{ }^{13} \mathrm{C} \mathrm{NMR}\left(100 \mathrm{MHz}, \mathrm{CDCl}_{3}\right): \delta-3.86,24.95,83.93,124.72$, 126.16, 127.10, 130.00, 131.67, 138.20, 140.09, 151.58, 158.89. ${ }^{29}$ Si NMR (79.3 MHz, $\left.\mathrm{CDCl}_{3}\right): \delta$ 5.21. HRMS(FAB): $646.3293\left(\mathrm{M}+\mathrm{H}^{+}\right)$. Calcd for $\mathrm{C}_{38} \mathrm{H}_{49} \mathrm{~B}_{2} \mathrm{O}_{4} \mathrm{Si}_{2}$ : 646.3277.

5e: Mp. 219-221 ${ }^{\circ} \mathrm{C} . \quad{ }^{1} \mathrm{H} \mathrm{NMR}\left(400 \mathrm{MHz}, \mathrm{CDCl}_{3}\right): \delta 0.47(\mathrm{~s}, 12 \mathrm{H}), 7.33$ (t, $J=7.6 \mathrm{~Hz}$, 2H), $7.48(\mathrm{~s}, 4 \mathrm{H}), 7.49(\mathrm{t}, J=7.6 \mathrm{~Hz}, 2 \mathrm{H}), 7.54(\mathrm{~d}, J=7.6 \mathrm{~Hz}, 2 \mathrm{H}), 7.78(\mathrm{~d}, J=7.6 \mathrm{~Hz}$, 2H). ${ }^{13} \mathrm{C} \mathrm{NMR}\left(67.8 \mathrm{MHz}, \mathrm{CDCl}_{3}\right): \delta$-3.62, 124.93, 127.77, 127.88, 130.35, 131.12, 131.39, 136.54, 137.53. 143.79, 147.66. $\operatorname{HRMS(EI):~549.9767~}\left(\mathrm{M}^{+}\right)$. Calcd for $\mathrm{C}_{26} \mathrm{H}_{24} \mathrm{Br}_{2} \mathrm{Si}_{2}: 549.9783$.

5f: Mp. 218-220 ${ }^{\circ} \mathrm{C} . \quad{ }^{1} \mathrm{H}$ NMR (400 MHz, $\left.\mathrm{CDCl}_{3}\right): \delta 0.47(\mathrm{~s}, 12 \mathrm{H}), 6.81-6.88(\mathrm{~m}, 2 \mathrm{H})$, $6.86(\mathrm{~s}, 4 \mathrm{H}), 7.26-7.35(\mathrm{~m}, 4 \mathrm{H}), 7.62(\mathrm{~m}, 2 \mathrm{H}) .{ }^{13} \mathrm{C} \mathrm{NMR}\left(67.8 \mathrm{MHz}, \mathrm{CDCl}_{3}\right): \delta-3.83$, $122.50,126.91,127.40,130.22,132.08,137.19,137.33,137.73,147.75,150.99$. HRMS(FAB): $726.1273\left(\mathrm{M}+\mathrm{H}^{+}\right) . \quad$ Calcd for $\mathrm{C}_{38} \mathrm{H}_{25} \mathrm{~F}_{10} \mathrm{Si}_{2}: 726.1257$.

7a: Mp. 253-255 ${ }^{\circ} \mathrm{C} . \quad{ }^{1} \mathrm{H} \mathrm{NMR}\left(400 \mathrm{MHz}, \mathrm{CDCl}_{3}\right): \delta 0.51(\mathrm{~s}, 12 \mathrm{H}), 7.26(\mathrm{~m}, 2 \mathrm{H}), 7.38$ $(\mathrm{m}, 4 \mathrm{H}), 7.48-7.54(\mathrm{~m}, 6 \mathrm{H}), 7.60(\mathrm{~s}, 2 \mathrm{H}) .{ }^{13} \mathrm{C}$ NMR $\left(100 \mathrm{MHz}, \mathrm{CDCl}_{3}\right): \delta-3.14$, $126.44,126.97,127.73,128.77,139.27,140.81,141.27,144.84,147.60 .{ }^{29} \mathrm{Si} \mathrm{NMR}$ (79.3 MHz, $\mathrm{CDCl}_{3}$ ): $\delta$ 3.60. Anal. Calcd for $\mathrm{C}_{26} \mathrm{H}_{26} \mathrm{Si}_{2}: \mathrm{C}, 79.13 ; \mathrm{H}, 6.64$. Found: $\mathrm{C}$, 78.97, H, 6.64 .

7f: Mp. $>300{ }^{\circ} \mathrm{C} . \quad{ }^{1} \mathrm{H}$ NMR $\left(400 \mathrm{MHz}, \mathrm{CDCl}_{3}\right): \delta 0.45(\mathrm{~s}, 12 \mathrm{H}), 6.98(\mathrm{~m}, 4 \mathrm{H}), 7.06(\mathrm{~s}$, 
2H), 7.17-7.26 (m, 6H). ${ }^{13} \mathrm{C}$ NMR (67.8 $\left.\mathrm{MHz}, \mathrm{CDCl}_{3}\right): \delta-4.10,125.82,126.59$, 126.85, 128.49, 139.00, 140.12, 147.06, 152.22. HRMS (EI): $726.1245\left(\mathrm{M}+\mathrm{H}^{+}\right)$. Calcd for $\mathrm{C}_{38} \mathrm{H}_{25} \mathrm{~F}_{10} \mathrm{Si}_{2}: 726.1257$.

9: $\mathrm{Mp} 226-228{ }^{\circ} \mathrm{C} . \quad{ }^{1} \mathrm{H}$ NMR $(270 \mathrm{MHz}): \delta 0.47(\mathrm{~s}, 12 \mathrm{H}), 7.12(\mathrm{~s}, 2 \mathrm{H}), 7.19(\mathrm{t}, J=6.9$ $\mathrm{Hz}, 2 \mathrm{H}), 7.26(\mathrm{~d}, J=6.9 \mathrm{~Hz}, 2 \mathrm{H}), 7.32(\mathrm{t}, J=6.9 \mathrm{~Hz}, 2 \mathrm{H}), 7.52(\mathrm{~d}, J=6.9 \mathrm{~Hz}, 2 \mathrm{H}) . \quad{ }^{13}$ C NMR $\left(67.8 \mathrm{MHz}, \mathrm{CDCl}_{3}\right): \delta-2.93,124.01,126.53,129.97,131.73,138.30,143.50$, 146.51, 149.46. ${ }^{29} \mathrm{Si} \mathrm{NMR}\left(79.3 \mathrm{MHz}, \mathrm{CDCl}_{3}\right): \delta 2.23 . \quad \mathrm{HRMS}(\mathrm{EI}): 318.1254\left(\mathrm{M}^{+}\right)$. Calcd for $\mathrm{C}_{20} \mathrm{H}_{22} \mathrm{Si}_{2}: 318.1260$. 